\title{
Asymmetry of Wigner's time delay in a small molecule
}

\author{
Alexis Chacon, ${ }^{1, *}$ Manfred Lein, ${ }^{2}$ and Camilo Ruiz ${ }^{1}$ \\ ${ }^{1}$ Grupo de Investigación en Óptica Extrema, Universidad de Salamanca, E-37008 Salamanca, Spain \\ ${ }^{2}$ Institut für Theoretische Physik and Centre for Quantum Engineering and Space-Time Research (QUEST), \\ Leibniz Universität Hannover, Appelstraße 2, D-30167 Hannover, Germany
}

(Received 4 February 2014; published 29 May 2014)

\begin{abstract}
Ionization by an attosecond pulse launches an electron wave packet in the continuum which contains rich information about the pulse, the parent system, and the ionization dynamics. This emission process is not instantaneous in the sense that the electrons take a finite time to leave the potential. This time is closely related to the Wigner time. In this paper we introduce the stereo Wigner time delay, which measures the relative delay between electrons emitted to the left and right in an asymmetric system. We present a theoretical study of the delay in photoemission for a small asymmetric molecular system using the streaking technique. The stereo Wigner time delay shows advantages compared to previous schemes. Our numerical calculation shows that such a measurement removes the infrared laser-Coulomb coupling, which has been problematic in the interpretation of the measured delay in photoemission from atomic systems.
\end{abstract}

DOI: 10.1103/PhysRevA.89.053427

PACS number(s): $32.80 . \mathrm{Fb}, 32.80 . \mathrm{Rm}, 42.50 . \mathrm{Hz}$

\section{INTRODUCTION}

During the last two decades, advances in laser technology and the understanding of the nonlinear processes in laser-matter interaction have allowed us to produce extreme ultraviolet (XUV) pulses with extremely short duration below the femtosecond scale. Attosecond pulses are a unique tool to study electronic quantum processes on their natural time scale $[1,2]$.

When a single attosecond pulse (SAP) or an attosecond pulses train interacts with an atom or a molecule, a coherent ultrabroadband electron wave packet (EWP) is created. If the photon energies in the attosecond pulse are higher than the ionization potential, the electron is freed, and the electron momentum distribution maps the characteristics of the attosecond pulse and the parent system [3-5]. These electrons are not emitted instantaneously. Instead, the atom or molecule may have a "response time" or "delay" in the photoemission [6]. Since the electron travels out of the binding potential with finite velocity, the delay is of the order of the atomic unit of time. It is related to the so-called Wigner time $[7,8]$, which measures the travel time difference between a free electron and an electron under the influence of a short-range potential. The response time of the atom or the molecule is encoded in the phase of the EWP and provides valuable information about the system [5,6]. But since the information is in the phase, traditional observables cannot access this quantity.

Only recently have observations of the delay in photoionization been carried out thanks to the now available tools of attoscience. Schultze and coworkers [9] have measured the relative delay in photoemission from the $2 s$ and $2 p$ subshells of neon using the streaking technique [10]. The measurement is based on the production of a SAP of some 200-as duration and a central energy of $106 \mathrm{eV}$ together with a short infrared (IR) laser pulse. The results showed a 21-as relative delay between the $2 s$ and $2 p$ orbitals. Also recently, the reconstruction of

*achacon@usal.es attosecond beating by interference of two-photon transitions (RABBIT) technique [11] has been used to measure the relative delay between the $3 s$ and $3 p$ subshells in argon [12]. This technique uses an attosecond pulse train with a mean energy of $35 \mathrm{eV}$ in the presence of a moderate IR laser pulse. In this case, the $3 p$ electron shows an apparent delay of some 100 as relative to the $3 s$ electron, which seems to leave the atom earlier.

Several papers have addressed the relation between the measured times and the intrinsic delay in photoionization or Wigner time [13,14]. While the Wigner time is included in the measured time, some other factors such as the polarization of the initial state [14], multielectron effects [15], and, more important, the laser-Coulomb coupling are included in the experimental observable $[12,14,16]$. Recent work has also theoretically addressed the time delay in small molecules such as hydrogen molecules [13] and other two-center molecules [17], emphasizing the consequences of having two centers.

In this paper we analyze the delay in photoemission for an asymmetric molecule and focus on the left-right asymmetries of this delay. We term the left-right time difference the stereo Wigner time delay (SWTD). There are certain advantages of using this quantity. First of all, a single orbital is analyzed, which means that it is not necessary to analyze two different orbital shapes and different binding energies. Second, the problem of the laser-Coulomb coupling in the streaking or RABBIT techniques is removed with the stereo measurement. Due to the symmetry of the long-range contribution, it is removed from the measurement with the left-right SWTD definition.

In Sec. II, we introduce a one-dimensional (1D) model for a small oriented two-center molecule with properties similar to the carbon monoxide (CO) molecule. We compute the SWTD from the exact dipole matrix element and describe the results expected from stereo measurements. In Sec. III, we compare the results from the dipole matrix element to numerical results for the travel time for the left and right electrons. Finally, in Sec. IV, we simulate the streaking technique to extract the SWTD from experimental observables, and we comment on the robustness of the technique. 


\section{WIGNER TIME DELAY FOR AN ASYMMETRIC MOLECULE}

Similar to the group delay in ultrafast optics, the delay in photoemission can be defined as the energy derivative of the photoionization scattering phase shift, i.e., the phase of the dipole matrix element between the initial state and the final continuum state [6]. Wigner introduced the delay between a plane wave propagating freely and the continuum state in an atomic potential [7,8]. If the angular momentum $l$ is well defined, this time is directly related to the scattering phase $[18,19]$. In the case of photoionization from a single state, the delay in photoionization can be defined as the derivative $\Delta t_{\mathrm{W}}=\frac{\partial \phi_{l}(E)}{\partial E}$ of the dipole matrix element phase $\phi_{l}(E)$ for transition between the initial state and the continuum state with respect to the energy $E$. For brevity we will refer to this quantity as Wigner time delay.

Next, we introduce a model for an oriented asymmetric molecule with properties similar to those of $\mathrm{CO}$, and we compute the exact dipole matrix element to extract the SWTD. A solution of the full time-dependent Schrödinger equation (TDSE) for the three-dimensional molecule is very demanding as it involves several degrees of freedom for the nuclei and the electrons. We therefore introduce a 1D two-center model with a single active electron. It is similar to those used in the literature [20] for $\mathrm{CO}$. We fix the positions of the nuclei as their dynamics is much slower than the electron dynamics. In exchange, this model allows us to calculate the exact continuum states and the complex dipole matrix element, from which the Wigner time can be calculated directly.

The field-free Hamiltonian for the 1D model is $H=\frac{p^{2}}{2}+$ $V(z)$. Atomic units are used throughout the paper. To mimic an oriented $\mathrm{CO}$ molecule along the laser polarization axis, we define the potential $V_{\mathrm{M}}(z)$ and compare it to the hydrogen atom with soft-core potential $V_{\mathrm{H}}(z)$.

$$
\begin{gathered}
V_{\mathrm{H}}(z)=-\frac{1}{\sqrt{a_{0}+z^{2}}}, \\
V_{\mathrm{M}}(z)=-\frac{Z_{1}}{\sqrt{a_{1}+\left(z-R_{1}\right)^{2}}}-\frac{Z_{2}}{\sqrt{a_{2}+\left(z-R_{2}\right)^{2}}} .
\end{gathered}
$$

To solve the time-independent Schrödinger equation we use the code QFISHBOWL [21], which implements the split-operator method [22] in one, two, and three dimensions. The ground states are obtained using imaginary-time propagation. The grid parameters are the same in both systems, and the time step for the ground-state calculation is $\Delta t=-0.01 i$. The grid size is 2500 a.u. with a spacing $\Delta z=0.1$ a.u. For $\mathrm{H}$ the soft-core parameter is $a_{0}=2$ a.u., which yields a ground state with ionization potential $I_{p}=0.5$ a.u. For the oriented molecule model we use the soft-core parameters $a_{1}=1.60, a_{2}=1.33$, the charges $Z_{1}=0.67, Z_{2}=0.33$, and the core positions $R_{1}=-0.6$ a.u., $R_{2}=1.65$ a.u. These parameters are chosen such that the ground-state energy matches the ionization potential $I_{p}=0.5$ a.u. and that the internuclear distance is $R=$ 2.25 a.u., which are close to the values of $\mathrm{CO}$ [23]. The choice of $Z_{1}$ and $Z_{2}$ is motivated by the fact that an electron far away from the center should move in an asymptotic potential of $-1 /|z|$, i.e., $Z_{1}+Z_{2}=1$. Furthermore, we choose $Z_{1} \approx 2 Z_{2}$, where $Z_{1}$ refers to $O$ and $Z_{2}$ refers to $C$, accounting roughly for the positive charge being greater on the screened $\mathrm{O}$ than on the screened $\mathrm{C}$ in a CO molecule [24]. The potential is positioned such that the maximum of the electron density is located close to the origin. Our results below confirm that this choice leads to excellent agreement between the Wigner time delay and the streaking time delay. Note that a shift of the origin affects the Wigner time delay and also the time of flight discussed below, while the streaking time delay is independent of such a shift.

The 1D model for the molecule allows us to calculate the exact continuum functions. These are constructed by numerically matching states in the grid to the asymptotic Coulomb wave $\Phi_{p}^{(a)}(z)=\frac{1}{\sqrt{2 \pi}} \exp \left[i p z+i \frac{Z}{|p|} \ln (2 p z)\right]$ for $z \rightarrow \infty \operatorname{sgn}(p)[5,25]$. They can be efficiently computed for all values of the final momentum $p$. The momentum grid size is 10 a.u., and the momentum step is $\Delta p=0.01$ a.u. From these states, we calculate the complex dipole transition bound-free matrix element $d(p)=-\left\langle\Psi_{p}|z| \Psi_{0}\right\rangle$ from the initial state $\left|\Psi_{0}\right\rangle$ to the continuum state $\left|\Psi_{p}\right\rangle$. We then extract the Wigner time $\Delta t_{\mathrm{W}}$ according to the definition. We compute also the dipole matrix element using plane waves for comparison. If the continuum states were obtained by matching the grid solutions to plane waves, the Wigner time would not converge as the matching point is moved to infinity.

The projections on the plane waves and the scattering waves give different absolute dipole amplitudes in the $\mathrm{H}$ system [see Figs. 1(a) and 1(b)]. The phases shown in Figs. 1(a) and 1(b) also differ significantly. For the $\mathrm{CO}$ system, the dipole amplitudes calculated using both methods differ, as shown in Figs. 1(c) and 1(d). For the plane-wave projection, the CO dipole amplitude is symmetric, while it is slightly asymmetric for the scattering waves. The green dashed lines in Figs. 1(c) and 1(d) show that the dipole phases also differ strongly due to the influence of the Coulomb potential. The asymmetry of the $\mathrm{CO}$ potential is exposed in the dipole matrix element, which in turn is mapped into the electrons emitted to the left and the right after the absorption of an attosecond pulse. While the asymmetry occurs both in the dipole matrix element amplitude and phase, the asymmetry in the amplitude is very small and is probably very hard to measure. On the contrary, the asymmetry in the dipole phase is large and therefore sensitive to the details of the asymmetric potential. It is a more powerful tool to measure the characteristics of the molecule.

We define the left Wigner time as the derivative of the dipole phase with respect to the energy for electrons with negative momentum $\Delta t_{\mathrm{W}}^{(\mathrm{L})}=\frac{1}{p} \frac{\partial \phi(p)}{\partial p}$. The right Wigner time is defined as $\Delta t_{\mathrm{W}}^{(\mathrm{R})}=\frac{1}{p} \frac{\partial \phi(p)}{\partial p}$ for electrons with positive momentum, and the SWTD is defined as the difference between these two quantities, $\Delta t_{\mathrm{W}}^{(\mathrm{LR})}=\Delta t_{\mathrm{W}}^{(\mathrm{L})}-\Delta t_{\mathrm{W}}^{(\mathrm{R})}$. For the $\mathrm{H}$ atom and molecular system, Fig. 2 shows the SWTD. Both the plane waves and scattering waves yield a SWTD of zero in the atomic case. However, for the molecular case the SWTD is not zero. A clear minimum is obtained, which changes its position depending on whether plane waves or scattering waves are used. In Fig. 2(d) we plot the relative asymmetry of the dipole amplitude $S_{A}(E)=\frac{\left|d_{\mathrm{L}}(E)\right|-\left|d_{\mathrm{R}}(E)\right|}{\left|d_{\mathrm{L}}(E)\right|+\left|d_{\mathrm{R}}(E)\right|}$ as a function of the photoelectron energy $E=\frac{p^{2}}{2}$. We have found that $S_{A}(E)$ is very small and thus difficult to measure in an experiment. 


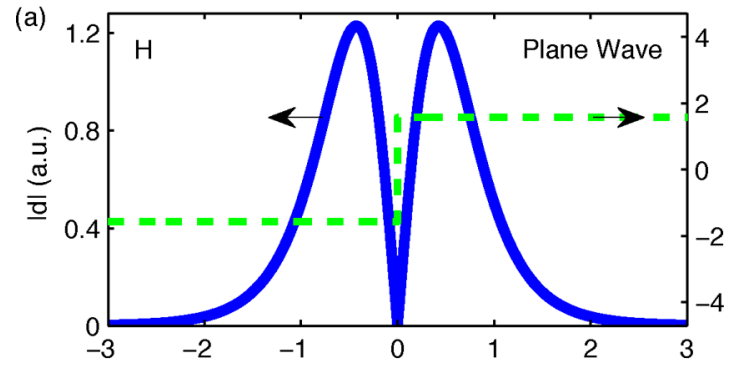

(b)
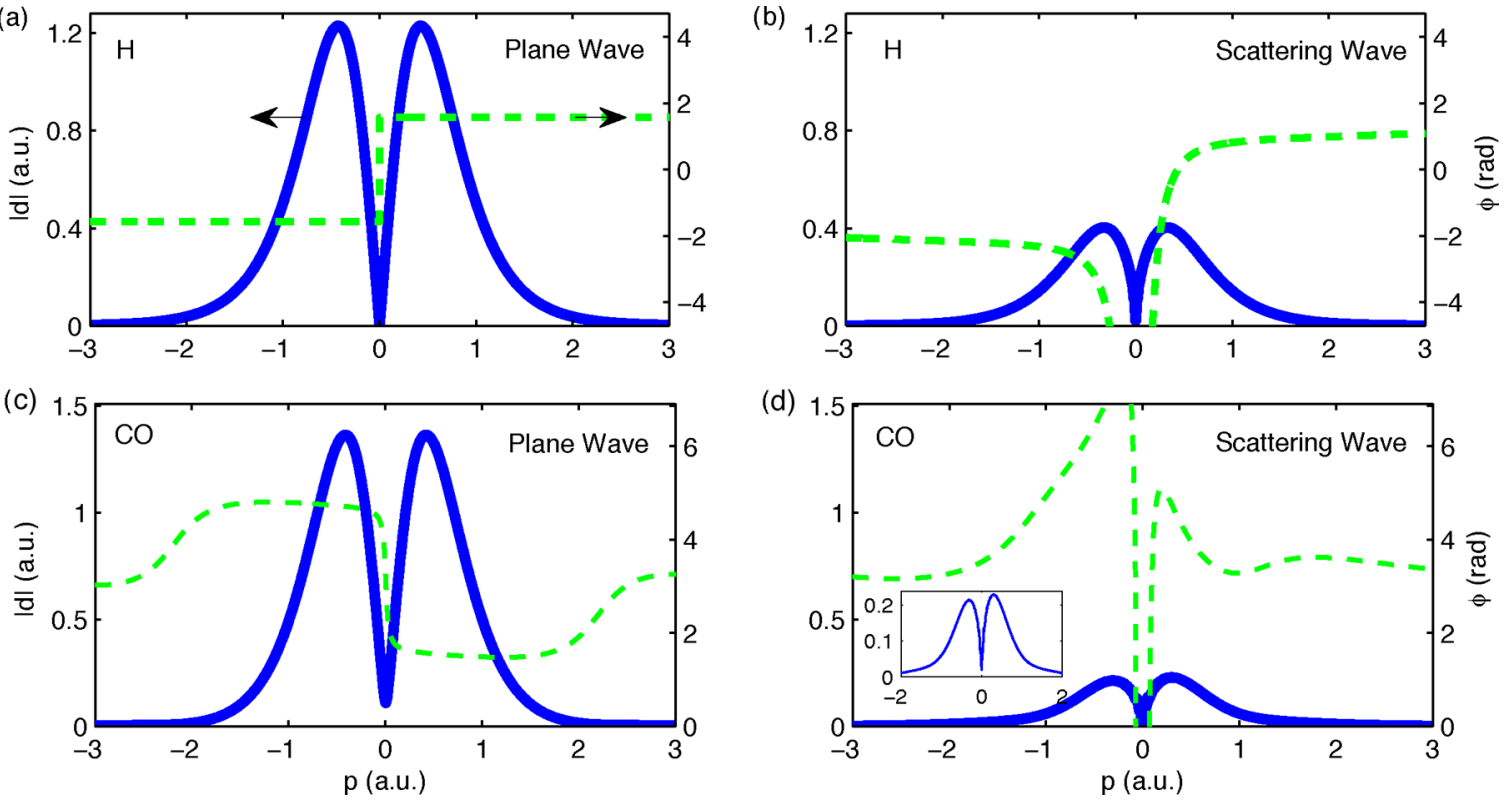

(d)

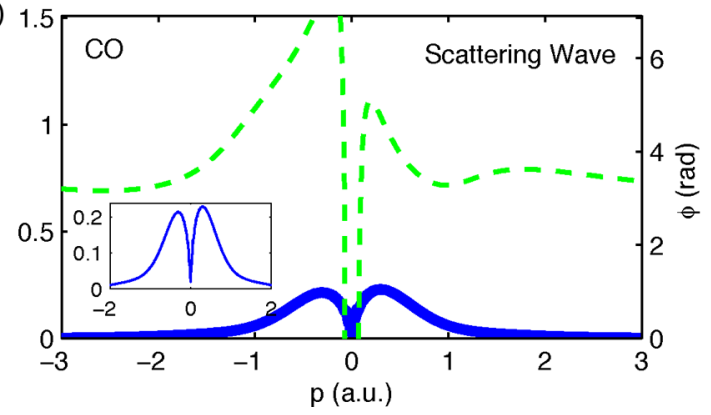

FIG. 1. (Color online) Dipole matrix elements. The amplitude (blue solid line) and phase (green dashed line) of the dipole $d(p)$ for 1D H by projection on (a) plane and (b) scattering waves. (c) and (d) The same as (a) and (b) but for a 1D CO molecular system. The inset graph depicts a zoom of the dipole amplitude, which demonstrates a small asymmetry in the case of scattering-wave calculation.

\section{TIME OF FLIGHT}

The SWTD can also be measured by tracking in time the EWPs emitted to either side. In this section, we estimate these times from a dynamical simulation, and we compute the asymmetry and compare it to the results obtained with the previous definition. We define the time $\Delta t_{\mathrm{TOF}}=t_{d}-t_{0}$ that an EWP spends in the continuum from an initial time $t_{0}$ until the arrival $t_{d}$ at a certain position $z_{d}$ as the "time of flight" (TOF). By taking the left-right difference, we will show that the SWTD can be obtained from the TOF method for the ionization induced by XUV attosecond pulses.
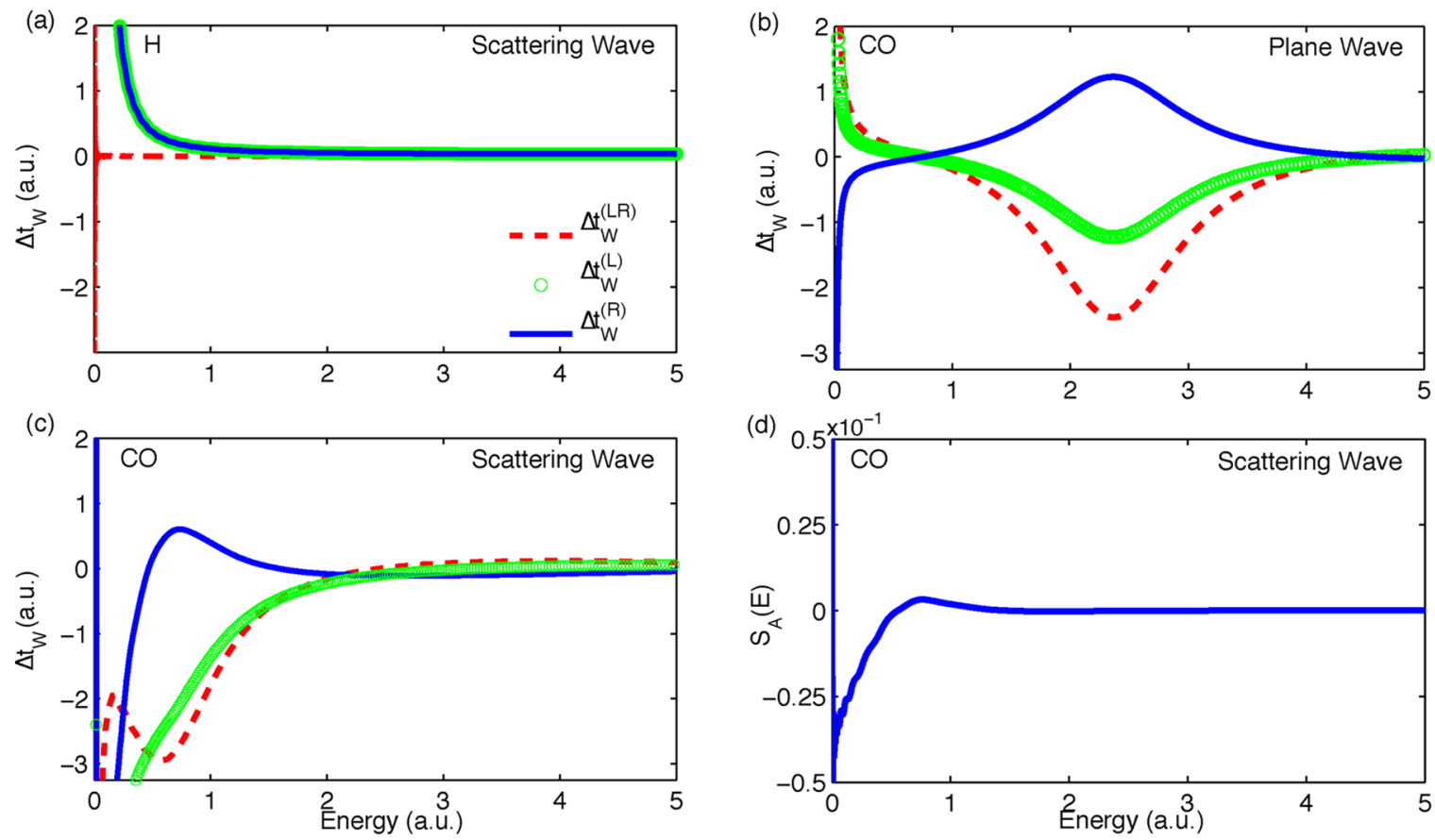

FIG. 2. (Color online) Stereo Wigner time with plane and scattering waves. (a) The left (green circles) and right (blue solid line) Wigner times and the SWTD (red dashed line) $\Delta t_{\mathrm{W}}^{(\mathrm{LR})}$ in the H atom calculated by scattering waves. (b) and (c) Same as (a) but using plane and scattering waves for the asymmetric molecule CO, respectively. The minimum in the SWTD shifted in these two pictures. (d) The asymmetry of the dipole matrix element amplitude shows very small values in the molecular case. 

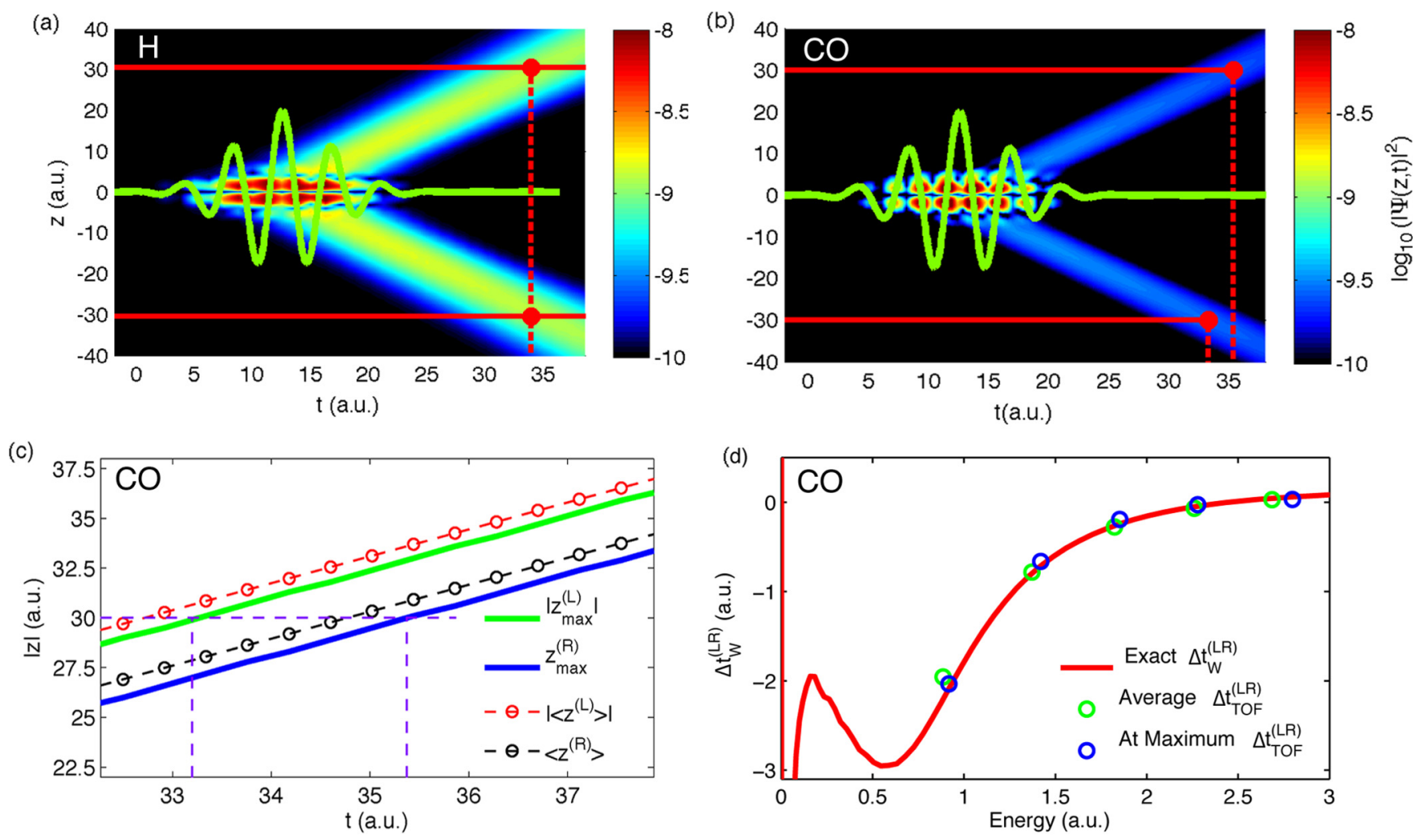

FIG. 3. (Color online) Ionization by a single attosecond pulse. Electron densities as a function of the position $z$ and time $t$ for the ionization by an XUV attosecond pulse are shown for (a) the atomic and (b) molecular systems. The red horizontal lines are the symmetric positions on either side $z_{d}= \pm 30$ a.u., and the red dashed lines are the corresponding arrival TOFs. (c) Absolute values of the positions at the maxima of electron densities [green (light gray) and blue (dark gray) solid lines] and the average values [red (gray) and black circles with dashed lines] as a function of time. The horizontal dashed line indicates the target position 30 a.u., and the vertical ones mark the corresponding TOFs for both sides. (d) Comparison between the stereo delay from the TOF technique using (i) the position of maximal density [blue (dark gray) circles] and (ii) average position values [green (light gray) circles] and the results extracted from the exact dipole phase as a function of the photoelectron energy.

The emission of EWPs on both sides by the absorption of a SAP are tracked in time, as shown in Figs. 3(a) and 3(b). We consider symmetric final positions on the left and right $\left(\left|z_{d}^{(\mathrm{L})}\right|=\left|z_{d}^{(\mathrm{R})}\right|\right)$. The EWPs take times $\Delta t_{\mathrm{TOF}}^{(\mathrm{L})}$ and $\Delta t_{\mathrm{TOF}}^{(\mathrm{R})}$ to reach these positions. The difference $\Delta t_{\mathrm{TOF}}^{(\mathrm{LR})}=\Delta t_{\mathrm{TOF}}^{(\mathrm{L})}-\Delta t_{\mathrm{TOF}}^{(\mathrm{R})}$ can be understood as the relative delay between an EWP emitted to the left and another one to the right. We refer to these calculations as the stereo TOF delay.

The Hamiltonian of the system is $H(t)=\frac{1}{2}\left[p+A_{X}(t)\right]^{2}+$ $V_{\mathrm{M}}(z)$ in the velocity gauge, where $p$ denotes the electron momentum and $A_{X}(t)$ is the vector potential, defined as $A_{X}(t)=-\int^{t} d t^{\prime} E_{X}\left(t^{\prime}\right)$, with $E_{X}(t)$ being the electric field, which is linearly polarized along the molecular axis. The grid parameters are the same as in the last section, and the time step is $\Delta t=0.01$ a.u. The peak intensity of the attosecond pulse is $I_{X}=10^{12} \mathrm{~W} / \mathrm{cm}^{2}$, the central frequency is $\omega_{X}=$ 1.5 a.u. $(40.8 \mathrm{eV})$, and the pulse has a FWHM of 9.53 a.u. (230 as), a Gaussian envelope, and zero carrier envelope phase (CEP).

For both systems, the emitted electronic densities are calculated as a function of time by projecting out the first two bound states from the wave function. Figure 3 shows the results. In the case of the $\mathrm{H}$ atom, both EWPs reach the positions \pm 30 a.u. at the same time on either side. As shown by red dots in Fig. 3(a), the arrival TOFs for both sides are $\Delta t_{\mathrm{TOF}}^{(\mathrm{L})}=\Delta t_{\mathrm{TOF}}^{(\mathrm{R})}=34$ a.u. However, in the molecular case, the arrival TOFs are not the same, as shown in Fig. 3(b). The molecular values are $\Delta t_{\mathrm{TOF}}^{(\mathrm{L})}=33.27$ a.u. and $\Delta t_{\mathrm{TOF}}^{(\mathrm{R})}=$ 35.35 a.u. This gives a stereo TOF delay of $\Delta t_{\mathrm{TOF}}^{(\mathrm{LR})}=-2.08$ a.u., which is shown in Fig. 3(c). For this calculation we have tracked the positions of the EWP maxima $\left(z_{\text {max }}^{(\mathrm{L})}, z_{\text {max }}^{(\mathrm{R})}\right)$ and the average positions $\left[\left\langle z^{(\mathrm{L})}\right\rangle=\int_{-\infty}^{0} \Psi^{*}(z) z \Psi(z) d z,\left\langle z^{(\mathrm{R})}\right\rangle=\right.$ $\left.\int_{0}^{\infty} \Psi^{*}(z) z \Psi(z) d z\right]$ as a function of time $t$. The absolute values of these quantities are shown in Fig. 3(c) for the molecular case. The stereo TOF delay using the average position method gives $\Delta t_{\mathrm{TOF}}^{(\mathrm{LR})}=-2.00$ a.u., which is very close to the value obtained from the position of the maximal electron density, $\Delta t_{\mathrm{TOF}}^{(\mathrm{LR})}=-2.08$ a.u.

The comparison between the exact stereo delay $\Delta t_{\mathrm{W}}^{(\mathrm{LR})}$ defined via the derivatives of the dipole phase and the results $\Delta t_{\text {TOF }}^{(\mathrm{LR})}$ from the TOF method is depicted in Fig. 3(d) as a function of the photoelectron energy. The XUV attosecond pulse parameters for these calculations are $\omega_{X}=1.5,2.0,2.5$, 3.5 a.u. and FWHM = 9.5, 7.2, 5.7, 4.8, 4.1 a.u., respectively. The other parameters are the same as in the first example with 

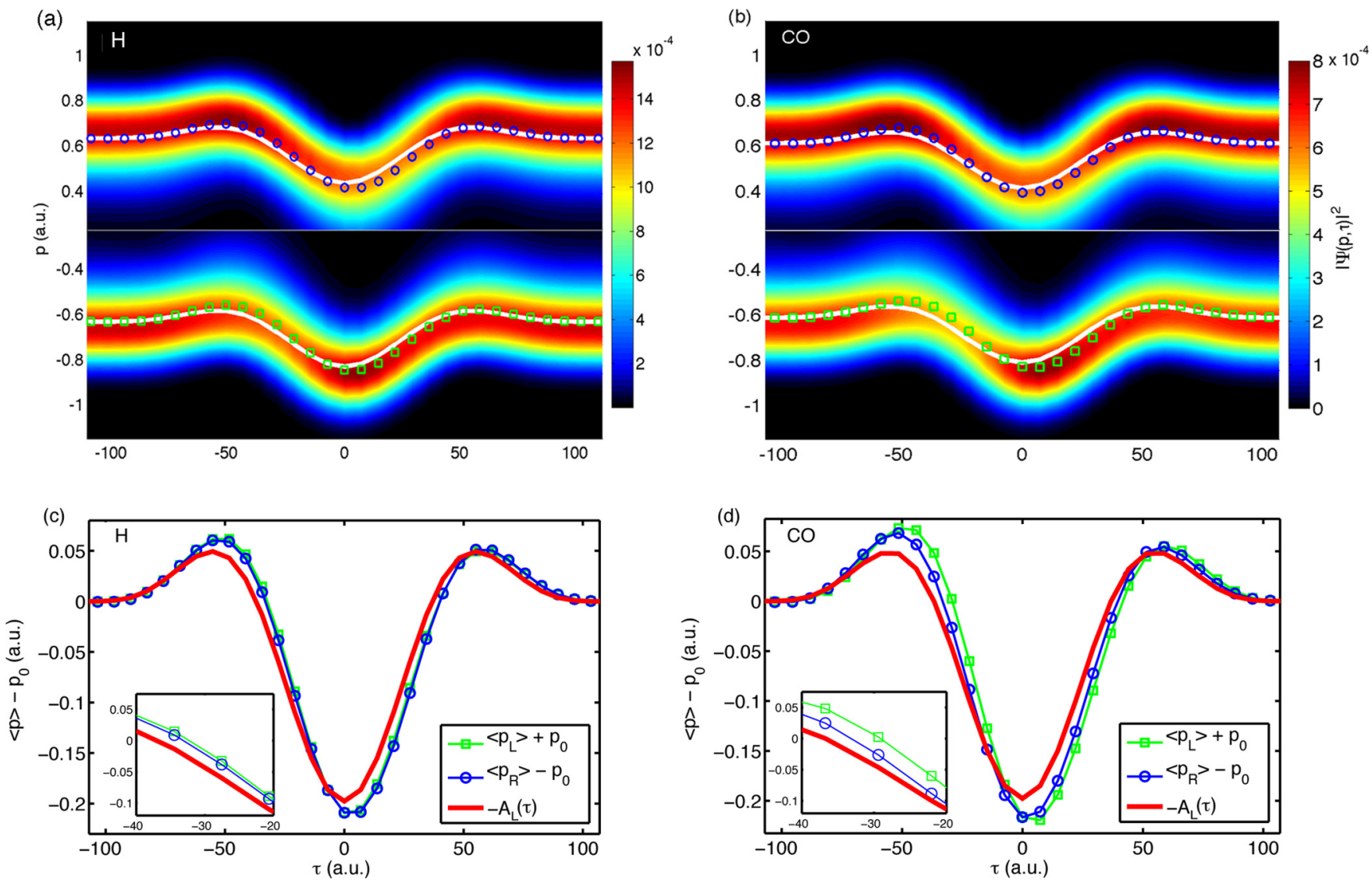

FIG. 4. (Color online) Streaking traces for measuring the stereo Wigner time delay. For H and CO, (a) and (b) show the streaking traces, which are the photoelectron momentum distributions as a function of time delay $\tau$ between the attosecond pulse and the IR laser field. White lines are the negative value of the vector potential, $-A_{\mathrm{L}}(\tau)$. The green squares and blue circles are the expectation values for the electrons with negative and positive momenta. These expectation values and the vector potential $-A_{\mathrm{L}}(\tau)$ for both systems are depicted in (c) and (d). The inset graph in (d) shows a clear time delay $\Delta t_{S}^{(\mathrm{LR})}=-2.9$ a.u. between electrons emitted to the left and right. As expected, in the case of the $\mathrm{H}$ atom, this difference is zero; see the inset in (c).

central frequency $\omega_{X}=1.5$ a.u. Both of the TOF methods give a very good agreement with the SWTD.

This method shows the physical meaning of the SWTD, but it is not suitable as a measurement scheme in the laboratory. In the following section we describe how to measure the SWTD with the streaking technique and the issues related to such a measurement.

\section{MEASUREMENT OF THE TIME DELAY BY THE STREAKING TECHNIQUE}

The streaking technique is a pump-probe technique which consists of the absorption of a SAP in the presence of a moderate and short IR pulse [2]. The final momentum of the electrons emitted is modified according to the instantaneous value of the vector potential at the time when the attosecond pulse acts. The final momentum is $p\left(\tau_{a}\right)=p_{0}-A_{\mathrm{L}}\left(\tau_{a}\right)$, where $\tau_{a}$ is the time at which the electron starts to feel the presence of the IR field. Here, $p_{0}$ is the central photoelectron momentum without IR field, $p_{0}=\sqrt{2\left(\omega_{X}-I_{p}\right)}$, which depends on the central XUV frequency $\omega_{X}$ and the ionization potential $I_{p}$. The Wigner time delay $\Delta t_{\mathrm{W}}$ is intuitively expected to shift the appearance time $\tau_{a}$. Experimental results by Schultze et al. [9] show a relative time delay in the photoemission from the $2 s$ and $2 p$ orbitals of the neon atom. However, the measured time using the streaking technique contains more than the Wigner

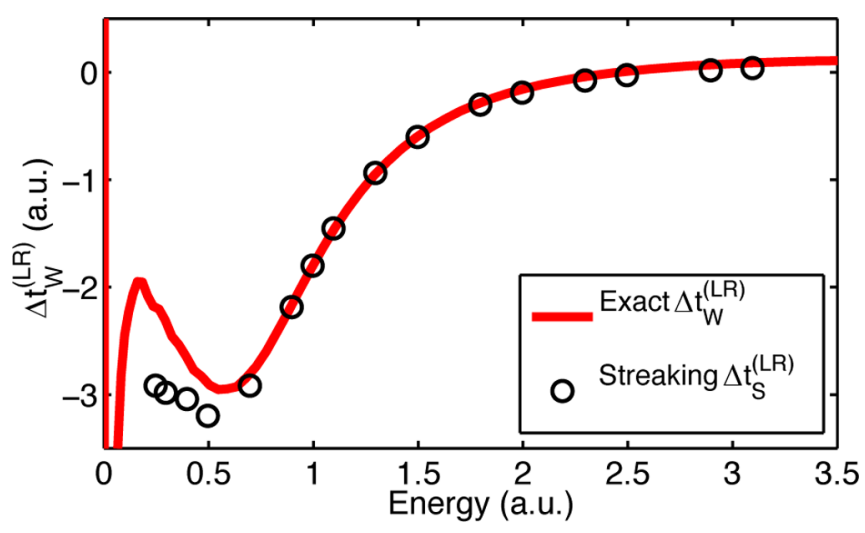

FIG. 5. (Color online) Streaking measurement of the stereo Wigner time delay. The graph shows the comparison between the exact SWTD $\Delta t_{\mathrm{W}}^{(\mathrm{LR})}$ (red line) and the time $\Delta t_{S}^{(\mathrm{LR})}$ obtained by the streaking technique (black circles) as a function of the photoelectron energy. 

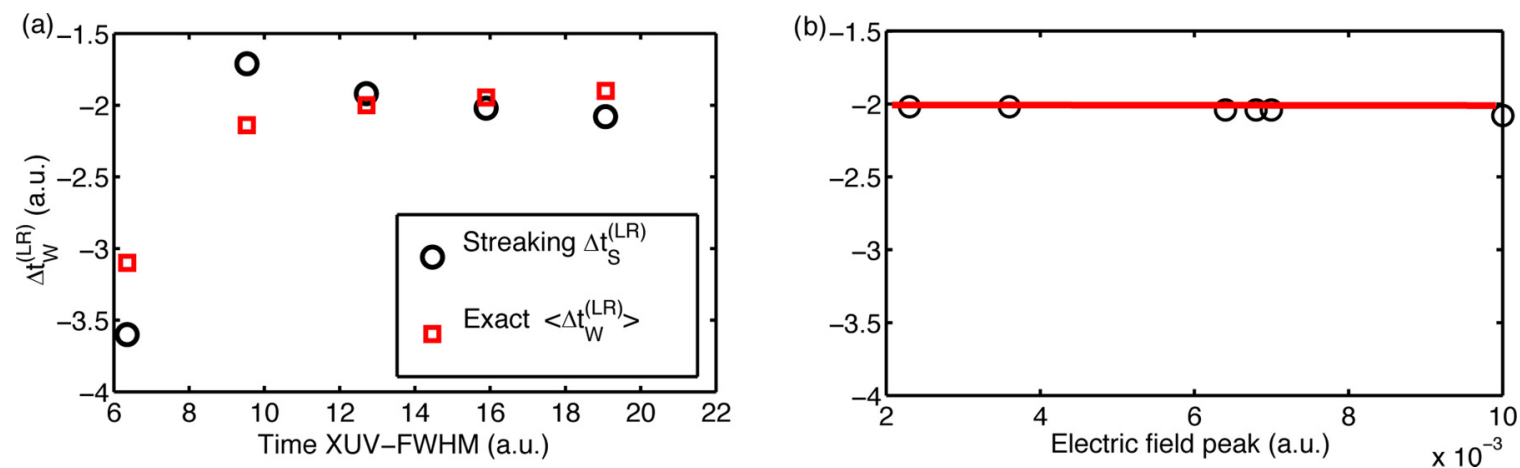

FIG. 6. (Color online) Stereo Wigner time delay for the molecular case. (a) Stereo Wigner time delay as a function of XUV FWHM. The red squares depict the averages of the exact SWTD $\left\langle\Delta t_{\mathrm{W}}^{(\mathrm{LR})}\right\rangle$ within the photoelectron energy bandwidth of the EWP. The black circles are the values measured by the streaking technique, $\Delta t_{S}^{(\mathrm{LR})}$. (b) SSTD as a function of the IR peak electric field strength (black circles).

time $[13,16]$. The basic assumption of the streaking technique is that the IR field modifies neither the initial state nor the final continuum state except for the momentum shift $-A_{\mathrm{L}}\left(\tau_{a}\right)$. However, while the IR laser field effects can be neglected in the initial state, in the continuum the coupling between the laser fields and the Coulomb potential well produces a delay which needs to be removed from the measured delay in order to obtain the Wigner time delay $[14,16]$. Although this may be possible, the dependence of the laser-Coulomb coupling on the laser parameters makes it difficult in practice. The SWTD $\Delta t_{\mathrm{W}}^{(\mathrm{LR})}$ removes the laser-Coulomb coupling from the measurement because it is identical on the left and on the right [16]. The SWTD avoids the need to estimate this contribution for each ionization channel. In turn, the SWTD can only be applied to asymmetric systems. Note that in our terminology, "laser-Coulomb coupling" refers to the effect of the asymptotic potential $-1 /|z|$, which is the same on both sides. Any left-right difference of the potential is merely in the short-range behavior. Such a difference is therefore incorporated in the SWTD.

As the absorption of a SAP leads to the emission of electrons on both sides, two streaking traces can be recorded, one on the left and another one on the right, and the stereo streaking time delay (SSTD) can be obtained from them. We compute the streaking traces using the TDSE, which allows us to simulate the interaction of a SAP in the presence of a weak IR field and to record the final electron momentum density at the end of the pulses. We do this for each delay $\tau$ between the SAP and IR field. Figures 4(a) and 4(b) show the streaking traces for $\mathrm{H}$ and $\mathrm{CO}$, respectively. The attosecond pulse parameters are intensity $I_{X}=10^{12} \mathrm{~W} / \mathrm{cm}^{2}$, central frequency $\omega_{X}=0.75$ a.u. $(20 \mathrm{eV})$, and FWHM = 21.6 a.u. (524 as). The field has a peak intensity $I_{\mathrm{IR}}=2.5 \times 10^{12} \mathrm{~W} / \mathrm{cm}^{2}$, the central frequency is $\omega_{\mathrm{IR}}=0.057$ a.u., the temporal width is $\mathrm{FWHM}=2.7 \mathrm{fs}$, and the CEP is set to zero. The grid parameters are the same as in the previous section. The IR FWHM is fixed to a single cycle to keep the time of the numerical calculation small.

To extract the delay in photoemission from the streaking traces, we measure the expectation values $\left\langle p_{\mathrm{L}}(\tau)\right\rangle$ and $\left\langle p_{\mathrm{R}}(\tau)\right\rangle$ for all delays $\tau$ between the SAP and the IR pulse. We calculate the Fourier transform (FT) of these expectation values and then extract the time delay in photoemission for both sides $\Delta t_{S}^{(\mathrm{L})}=$
$\frac{\phi_{S}^{(\mathrm{L})}\left(\omega_{0}\right)}{\omega_{0}}$ and $\Delta t_{S}^{(\mathrm{R})}=\frac{\phi_{S}^{(\mathrm{R})}\left(\omega_{0}\right)}{\omega_{0}}$ as the FT phase $\phi_{S}(\omega)$ evaluated at the central frequency $\omega_{0}$ of the IR laser vector potential divided by $\omega_{0}$. Then we compare the extracted relative delay for each side $\Delta t_{S}^{(\mathrm{LR})}=\Delta t_{S}^{(\mathrm{L})}-\Delta t_{S}^{(\mathrm{R})}$.

The results show a time shift between the IR vector potential $-A_{\mathrm{L}}(\tau)$ and the expectation values for $\left\langle p_{L}\right\rangle$ and $\left\langle p_{R}\right\rangle$ in the $\mathrm{H}$ atom [see Fig. 4(c)]. As expected, the SSTD is zero for this case. In contrast, the streaking results for the molecule show clearly that there is a time delay between the $\left\langle p_{\mathrm{L}}\right\rangle$ and $\left\langle p_{\mathrm{R}}\right\rangle$ curves [see Fig. 4(d)] which is different from zero. This value is $\Delta t_{S}^{(\mathrm{LR})}=-2.9$ a.u. at a photoelectron energy $\left(E_{0}=\omega_{X}-I_{p}\right)$ of 0.24 a.u.

The results shown in Fig. 4 are for a single XUV attosecond pulse. To test whether the streaking technique works in a broad range of XUV frequencies, we have calculated the streaking traces for a set of carrier frequencies between $0.75(20.40 \mathrm{eV})$ and 3.6 a.u. $(97.95 \mathrm{eV})$. The results are depicted in Fig. 5. They show that the retrieved SSTD is in very good agreement with the exact stereo Wigner time delay $\Delta t_{\mathrm{W}}^{(\mathrm{LR})}$ as obtained from the exact complex dipole matrix element and the TOF technique defined above. This shows that the SWTD can be measured experimentally and provides a simple way to remove the laser-Coulomb coupling. The technique is very sensitive to the asymmetry of the molecular potential and is robust to laser parameter changes.

We also address the natural question on how the SWTD changes with the temporal width of the attosecond pulse or with the peak intensity of the IR laser pulse, which determines the laser-Coulomb coupling. Figure 6(a) shows the average $\left\langle\Delta t_{\mathrm{W}}^{(\mathrm{LR})}\right\rangle=\frac{\int \Delta t_{\mathrm{W}}^{(\mathrm{LR})}(E)|\Psi(E)|^{2} d E}{\int|\Psi(E)|^{2} d E}$ of the stereo Wigner time as a function of the XUV attosecond pulse duration (FWHM). Here, $|\Psi(E)|^{2}$ is the energy density of the EWP. The attosecond pulse used in these simulations has a central frequency of $\omega_{X}=1.5$ a.u. and a peak intensity of $I_{X}=10^{12} \mathrm{~W} / \mathrm{cm}^{2}$, and the CEP is set to zero. The XUV attosecond pulse parameters used in Fig. 6(b) are the same as in the simulation results depicted in Fig. 6(a), but the FWHM is fixed to 12 a.u. This average is in good agreement with the results $\Delta t_{S}^{(\mathrm{LR})}$ from the streaking method. The streaking result $\Delta t_{S}^{(\mathrm{LR})}$ as a function of the IR peak electric field is constant [see Fig. 6(b)]. This 
demonstrates that the laser-Coulomb coupling has been effectively eliminated.

\section{CONCLUSIONS}

Attosecond pulses are a useful probe for the dynamics of ionization. In small oriented asymmetric molecules, the Wigner time delay is different for left and right photoelectrons. Even when the dipole amplitude asymmetry is small, as in the case presented here, the stereo Wigner time delay is significant and provides information about the dipole transition matrix element phase. We have shown that the SWTD can be measured by the streaking technique, and it is not affected by the unwanted laser-Coulomb coupling. The method is robust against variation of the frequency, duration of the pulse, and streaking field intensity.

\section{ACKNOWLEDGMENTS}

This research was funded by the Spanish Ministerio de Ciencia e Innovación (MICINN), through Consolider Program SAUUL CSD2007-00013 and research project FIS200909522. Support from the Centro de Láseres Pulsados (CLPU) is also acknowledged. A.C. thanks Secretaría Nacional de Ciencia Innovación y Tecnología (SENACYT) Panama for the financial support. C.R. thanks the program Ramón y Cajal.
[1] P. B. Corkum and F. Krausz, Nat. Phys. 3, 381 (2007).

[2] F. Krausz and M. Ivanov, Rev. Mod. Phys. 81, 163 (2009).

[3] F. Quéré, Y. Mairesse, and J. Itatani, J. Mod. Opt. 52, 339 (2005).

[4] F. Quéré, J. Itatani, G. L. Yudin, and P. B. Corkum, Phys. Rev. Lett. 90, 073902 (2003).

[5] A. Chacon, M. Lein, and C. Ruiz, Phys. Rev. A 87, 023408 (2013).

[6] J. M. Dahlström, A. L'Huillier, and A. Maquet, J. Phys. B 45, 183001 (2012).

[7] E. P. Wigner, Phys. Rev. 98, 145 (1955).

[8] F. T. Smith, Phys. Rev. 118, 349 (1960).

[9] M. Schultze, M. Fiess, N. Karpowicz et al., Science 328, 1658 (2010).

[10] M. Hentschel, R. Kienberger, Ch. Spielman, G. A. Reider, N. Milosevic, T. Brabec, P. Corkum, U. Heinzmann, M. Drescher, and F. Krausz, Nature (London) 414, 509 (2001).

[11] P. M. Paul, E. S. Toma, P. Breger, G. Mullot, F. Augé, Ph. Balcou, H. G. Muller, and P. Agostini, Science 292, 1689 (2001).

[12] K. Klünder, J. M. Dahlström, M. Gisselbrecht et al., Phys. Rev. Lett. 106, 143002 (2011).

[13] A. S. Kheifets, I. A. Ivanov, and I. Bray, J. Phys. B 44, 101003 (2011).

[14] S. Nagele, R. Pazourek, J. Feist, K. Doblhoff-Dier, C. Lemell, K. Tőkési, and J. Burgdörfer, J. Phys. B 44, 081001 (2011).

[15] A. S. Kheifets and I. A. Ivanov, Phys. Rev. Lett. 105, 233002 (2010).

[16] M. Ivanov and O. Smirnova, Phys. Rev. Lett. 107, 213605 (2011).
[17] V. V. Serov, V. L. Derbov, and T. A. Sergeeva, Phys. Rev. A 87, 063414 (2013).

[18] J. Su, H. Ni, A. Becker, and A. Jaroń-Becker, Phys. Rev. A 88, 023413 (2013).

[19] J. Su, H. Ni, A. Becker, and A. Jaroń-Becker, Phys. Rev. A 87, 033420 (2013).

[20] Y. J. Chen, L. B. Fu, and J. Liu, Phys. Rev. Lett. 111, 073902 (2013).

[21] C. Ruiz and A. Chacon, QFISHBOWL library, http://code.google.com/p/qfishbowl/.

[22] M. D. Feit, J. A. Fleck, and A. Steiger, J. Comput. Phys. 47, 412 (1982).

[23] National Institute of Standards and Technology (NIST), Computational Chemistry Comparison and Benchmark DataBase, http://cccbdb.nist.gov/.

[24] If we consider that the atomic $1 s$ and $2 s$ electrons of $\mathrm{C}$ and $\mathrm{O}$ screen the respective nuclear charges in a $\mathrm{CO}$ molecule, the screened $\mathrm{O}$ carries four positive charges compared to two positive charges on $\mathrm{C}$. Assuming that this ratio of 2 is unchanged as the system is filled up with the remaining electrons, we obtain the estimate $Z_{1} \approx 2 Z_{2}$. Unlike the highest occupied orbital obtained in a three-dimensional quantum chemistry calculation [26], the 1D ground state used in this work has a dipole pointing from $\mathrm{O}$ to $\mathrm{C}$ even though $a_{2}<a_{1}$ is used in order to move the calculated orbital slightly into the direction of C.

[25] E. V. van der Zwan and M. Lein, Phys. Rev. Lett. 108, 043004 (2012).

[26] A. Etches and L. B. Madsen, J. Phys. B 43, 155602 (2010). 